\title{
Rib Locating on Chest Direct Radiography Image using Watershed Algorithm and Correlation Matching
}

\author{
Xuechen $\mathrm{Li}^{\mathrm{a}}$ Suhuai Luo ${ }^{\mathrm{a}}$ Qingmao $\mathrm{Hu}^{\mathrm{b}}$ \\ ${ }^{a}$ School of Design, Communication and IT, University of Newcastle, NSW, Australia \\ ${ }^{b}$ Research Lab for Medical Imaging and Digital Surgery, (Shenzhen Institutes of Advanced Technology, \\ Shenzhen, P. R. China)
}

\begin{abstract}
A rib locating method on chest direct radiography (DR) image using watershed algorithm and correlation matching is presented in this paper. Firstly, the body and spine are located by employing watershed algorithm; second, the body model is selected to remove other bones outside body; thirdly, the models of left and right ribs are resized and rotated to fit ribs of each side respectively; finally, the rib regions are extracted, each one of which contains only one rib. 70 DR images are used to test the method. The experiment result shows that the average error rate, accuracy, and sensitivity are respectively $0.067,0.828$ and 0.862 .
\end{abstract}

Keywords: rib locating; watershed algorithm; correlation match; chest DR image

\section{INTRODUCTION}

Automatic processing and recognition of biomedical images has become one of the most important branches of image processing. Much research efforts have been focused on CT and magnetic resonance images. Chest X-ray images, in contrast, are not so popular in recent years since it is not often used in surgery planning and navigation. However, it is still widely used in routine medical examination such as pulmonary tuberculosis and lung cancer. It is useful and significant to use computer technology and image processing to locate the lesion and help to make diagnosis. DR (direct radiography) is a newly developed medical imaging technology. Compared with traditional X-ray image, DR image is clearer, less time-consuming and easy to keep and save.

Rib locating is the first step of rib segmentation. It is important for rib lesion and lung disease diagnosis. There have been some works to deal with this problem [1-9]. Tomwaki et al. [1] used prior knowledge to design a weighted linear filter to enhance the ribs and binarize the image to locate the ribs. It is simple and fast, but strongly relies on the prior knowledge of body size and distance between ribs. Yue et al. [2] employed rule based Hough Transform to extract the rib borders. Quadratic curve was employed to approximating the curve of ribs. It is robust but time-consuming. Ginneken and Romeny [3] developed a model based method to segment ribs. They built a model with 10 parameters to describe different cases of ribs, 4 of them were described in detail: first one scales the height of ribs; second one adjusts the distance

2013 International Symposium on Computational Models for Life Sciences

AIP Conf. Proc. 1559, 90-97 (2013); doi: 10.1063/1.4825000

(C) 2013 AIP Publishing LLC 978-0-7354-1187-6/\$30.00 
between two neighbouring ribs; the third one shows the dissymmetry of left and right ribs; and the final one describes the thickness of ribs. This method processed all ribs as a whole object, deformed the model to fit the target. The advantage is that it will never miss any ribs; however, it may fall into local optimum and lead to mistaken result.

In this paper, we propose a rib locating method based on watershed algorithm and correlation matching. Section 2 presents the body and spine locating method on the basis of watershed algorithm and correlation matching; section 3 describes the rib locating method based on correlation matching; section 4 is the result of experiment and discussion; section 5 is conclusion and future work.

\section{BODY AND SPINE LOCATING METHOD}

Locating of body and spine is the first step of rib extraction. It gives the significant information such as body size, body border and spine location to guide rib locating. Based on this information, we can get rid of other bones like head or collarbone, estimate the size of ribs, and separate the image into left and right part to deal with left and right ribs respectively.

The body and spine locating method includes 2 main steps (see Fig. 1).

- The first step is to locate the left and right side of body and spine based on watershed algorithm. a) A 15 by 15 median filter was used to smooth the image $\left(\mathrm{I}_{\text {smooth }}\right)$; b) the watershed algorithm was used to find the potential location of body and spine; c) the morphological operation "open" was used in vertical direction to remove ribs and other noise; d) the longest 3 connect regions were selected as left side of body, spine and right side of body (see Fig. 2).

The second step is body shape estimation based on correlation matching. a) 3 different models were built to match the shape of body (see Fig. 3). The models are 3 types of average shape of body, they describe the different body width of different persons; b) the models are resized based on the body size information provided by watershed algorithm; c) The model which gets the highest match percentage is select as the body model of each image (see Fig. 4).

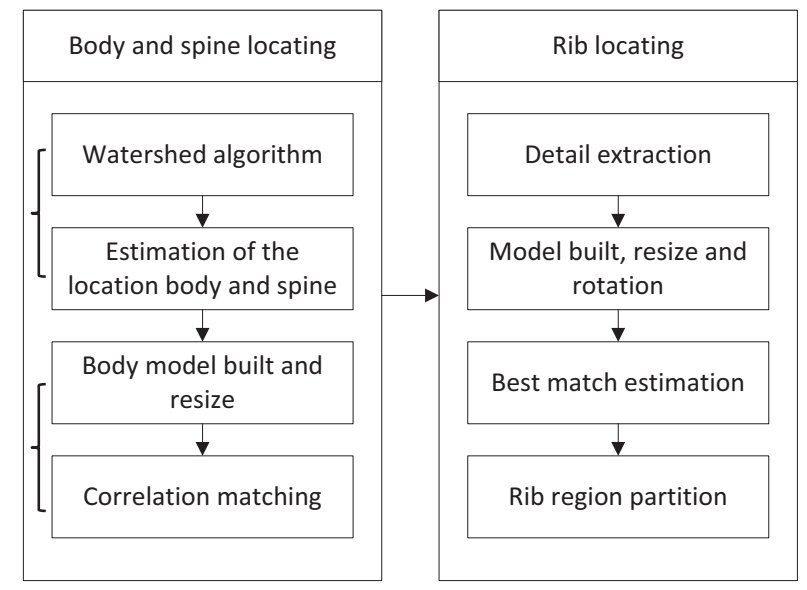

FIGURE 1. the over view of proposed rib locating method 

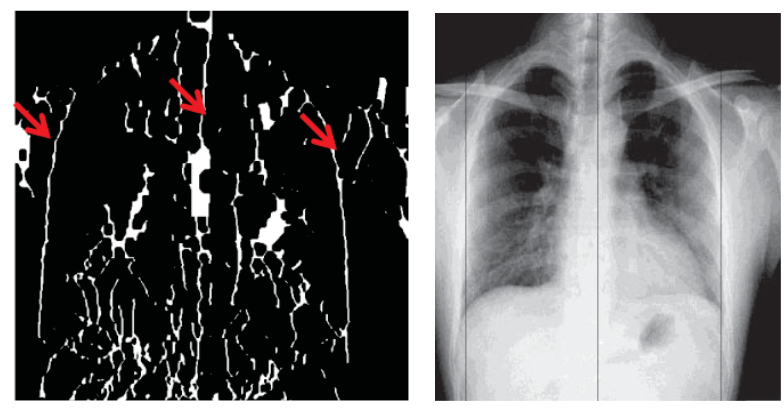

FIGURE 2. body and spine location using watershed algorithm
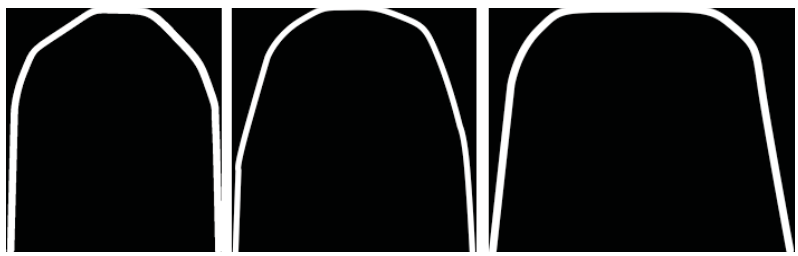

FIGURE 3. the body models

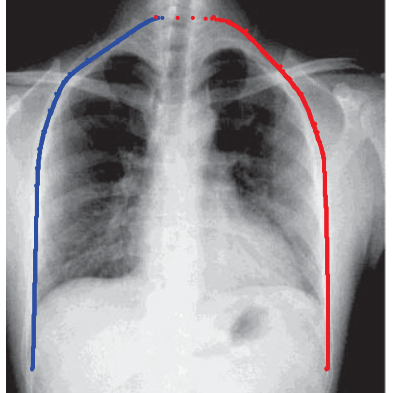

FIGURE 4. selected body model

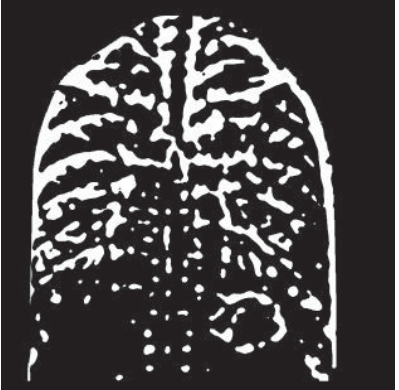

FIGURE 5. binaryzation of ribs

\section{Watershed algorithm}

Watershed algorithm is a mathematical morphological segmentation algorithm based on topology theory. The basic idea is to take the image as a topographic relief, where the grey level of a pixel is interpreted as its altitude in the relief, and every local minimum and its effect area is a catchment basin. The border of each catchment basin forms the watershed line.

One of the most common watershed algorithms was introduced by Meyer in 1994 [10]. The algorithm works on a gray scale image. During the successive flooding of the grey value relief, watersheds with adjacent catchment basins are constructed. This flooding process is performed on the gradient image, i.e. the basins should emerge along the edges. Normally this will lead to an over-segmentation of the image. To overcome the over-segmentation, either the image must be pre-processed or the regions must be merged on the basis of a similarity criterion afterwards. The main processing of watershed algorithm is as followed:

- A set of markers, pixels where the flooding shall start, are chosen. Each marker is given a different label. 
- The neighbouring pixels of each marked area are inserted into a priority queue with a priority level corresponding to the gray level of the pixel.

- The pixel with the highest priority level is extracted from the priority queue. If the neighbours of the extracted pixel that have already been labeled all have the same label, then the pixel is labeled with their label. All non-marked neighbours that are not yet in the priority queue are put into the priority queue.

- Repeat the above step until the priority queue is empty.

The non-labeled pixels are the watershed lines.

In our work, we employ Meyer's method but use the smoothed original image as the input rather than gradient image. The advantage is that the smoothed original image leads to less over-segmentation. The result of watershed algorithm only contains the midline of body border, spine, ribs and other bones. It is easy to get rid of ribs and other horizontal bones by employing morphological open operation in vertical direction.

\section{Correlation matching}

Template matching is one of the most popular image processing methods. Correlation matching is the simplest one, in some cases, also an effective one. The main idea of correlation matching is to select a sub-image (model), calculate the correlation between original image and the sub-image, and take the coordinate with highest correlation as the matching location. The correlation function is as followed:

$$
f(x, y) \circ w(x, y) \Leftrightarrow F(u, v) H^{*}(u, v)
$$

where $f(x, y)$ is the original image; $w(x, y)$ is the sub image; $F(u, v)$ is the Fourier transformation of $f(x, y) ; H(u, v)$ is the Fourier transformation of $w(x, y)$; "o" means correlation operation and “*” means complex conjugate.

In body locating step, we built 3 different models to describe the shape of body. Shown as Fig. 3, from left to right, the model describes wider body. The models are built based on the average body shape of chest X-ray images. Based on the location of left and right side of body which was estimated by watershed algorithm, we can resize the body model to match the size of body in different images. This makes our method more robust. The model with highest match percentage is selected as the body model. All pixels outside the model will be set to 0 so that only ribs remain in the image.

\section{RIB LOCATING METHOD}

Rib locating is the most important step before segmentation. A good locating method can make segmentation more accurate and robust. Similar to body locating method, correlation matching is also employed to locate ribs. The method includes 4 steps (see Fig. 1):

- The background of ribs is inhomogeneous. It is impossible to binarize ribs using a global threshold. To get the background, a 40 by 40 median filter was used to remove the details of the image ( $\left.I_{\text {remove detail }}\right)$; then we can get the image of detail: 


$$
\mathrm{I}_{\text {detail }}=\left|\mathrm{I}_{\text {smooth }}-\mathrm{I}_{\text {remove detail }}\right|
$$

After this step, the inhomogeneous background has been removed. The ribs and some noise (e.g. other organs under the lung) remain in the detail image; finally, a small threshold $(\mathrm{T}>0)$ was used to binarize $\mathrm{I}_{\text {detail }}$ (see Fig. 5);

- Two models were built to describe left and right ribs respectively (see Fig. 6). The models can be resized on the basis of the size of body; since the angles of ribs form different people are different, the angle of rib model must be changeable. It is ideal to use the angle as a prior knowledge but it is hard to estimate from the image directly. In our work, we let the angle change from 0 to 15 degree and select the best match case (the locations of ribs are homogeneous) as the final location of ribs.

In this work, similarity transformation is used to rotate the model:

$$
\begin{aligned}
& x=p \cos \theta-q \sin \theta \\
& y=p \sin \theta+q \cos \theta
\end{aligned}
$$

where $\mathrm{x}, \mathrm{y}$ are the coordinates of model after rotation, $\mathrm{p}, \mathrm{q}$ are the coordinates of model before rotation and $\theta$ is the angle of rotation.
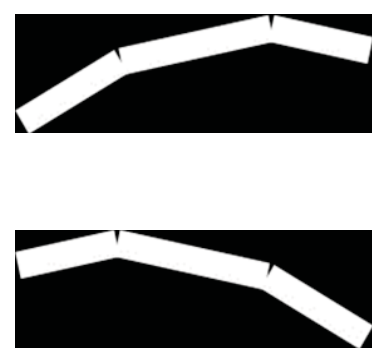

FIGURE 6. rib models

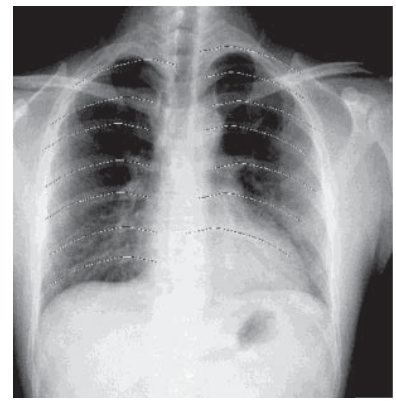

FIGURE 8. finally location of ribs
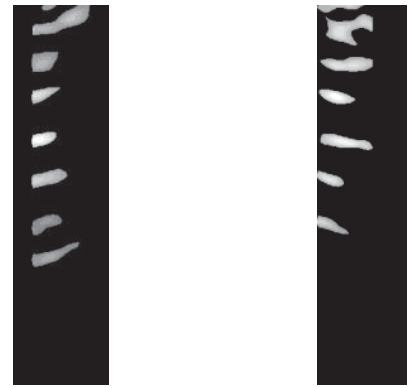

FIGURE 7. large correlations region

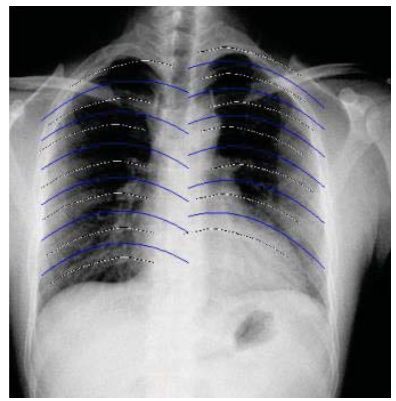

FIGURE 9. extracted rib area

When estimating the best match, a weighted parameter is used. In the best match cases, the locations of ribs are homogeneous. We defined a weighted parameter

$$
\mathrm{z}=\sqrt{\mathrm{k} \cdot \mathrm{x}^{2}+\mathrm{l} \cdot \mathrm{y}^{2}}
$$


where $\mathrm{x}, \mathrm{y}$ are the coordinates of ribs and $\mathrm{k}, \mathrm{l}$ are the weights. The values of parameters $\mathrm{k}$ and 1 are selected based on the experiment result. In our work, $\mathrm{k}=1$, $1=0.025$ when locating left ribs and $k=1,1=1$ when locating right ribs. The candidate whose $\mathrm{z}$ has the highest linearity is considered as the best match.

- The correlations between each rib model and its corresponding side of body were estimated respectively. The locations where correlations are large enough (larger than 0.7 largest correlations value) were selected (see Fig. 7). Each connected area is a potential rib location area. The brightness means the value of correlations of rib model and $\mathrm{I}_{\text {detail. }}$. The coordinates of largest correlation in each separate connected region were selected as the location of ribs (see Fig. 8).

- The average coordination of each pair of neighbouring ribs is the boundary of two separate rib area (see Fig. 9).

After these steps, the rib region could be extracted. In each region, there is only one rib. It is much more convenient to segment one rib in a small region than to do it in the whole image. Our work can make rib segmentation more accurate and efficient.

\section{RESULT AND DISCUSSION}

In our work, the method is realized by using Matlab and 70 different DR images are used to test our rib locating method. We use three metrics to evaluate the segmentation result: accuracy, sensitivity and error rate. The first one is used to evaluate the general performance of our method; the second one is used to evaluate the searching capability of rib locating methods and the third one focuses on the misjudgment of our method.

Accuracy is a widely used metrics to evaluate the performance of segmentation methods. It is defined as:

$$
\text { accuracy }=\frac{T P+T N}{T P+T N+F P+F N}
$$

TABLE 1. Performance metrics of the proposed rib locating algorithm

\begin{tabular}{cc}
\hline metrics & Mean value \\
\hline Accuracy & 0.828 \\
Sensitivity & 0.862 \\
Error rate & 0.067 \\
\hline
\end{tabular}

where TP is the number of true positive cases; TN is true negative cases; FP is false positive cases and $\mathrm{FN}$ is false negative cases.

Sensitivity is defined as:

$$
\text { sensitivity }=\frac{T P}{T P+F N}
$$

It means how many ribs are found in the outcome compare with ground truth.

Error rate is defined as:

$$
\text { error rate }=\frac{F P}{T P+F N}
$$


It means how many misclassified ribs have been selected according to our method.

Table 2 shows the experiment result.

To the best of our knowledge, this is the first reported results quantifying the location of ribs from X-ray images. The best match parameter $\mathrm{z}$ is adjusted to let the error rate as low as possible. However, it reduces the accuracy and sensitivity at the same time. The method can deal with most rib locating problems well, however, when the ribs are outside the lung area or ribs are not clear, the method will lose its effectiveness (see figure 10). Also it is time-consuming (about $50 \mathrm{~s}$ ) since every angle must be calculated when estimate the incline of ribs of both sides.

The reason of the incorrect locating is that: 1) the rib model can only be resized and rotated, but cannot be reshaped. The shapes of ribs are different even in one image. Using a fixed model to fit all ribs is not ideal; 2) the best match selected parameter $\mathrm{z}$ should be optimized. The $\mathrm{z}$ which is used in our method can reduce the error rate efficiently but cannot keep high accuracy and sensitivity at the same time; 3) when the ribs are out of lung area, they may be removed with the background; 4) when ribs are not clear in the original image, it is difficult to use a model to fit the image correctly.

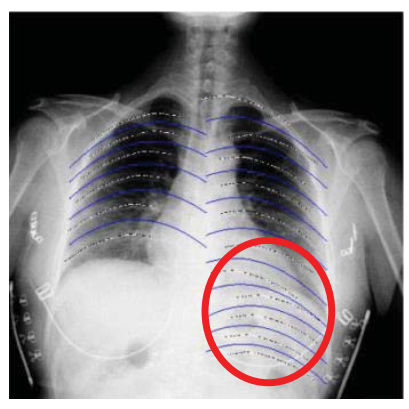

(a) ribs outside lung

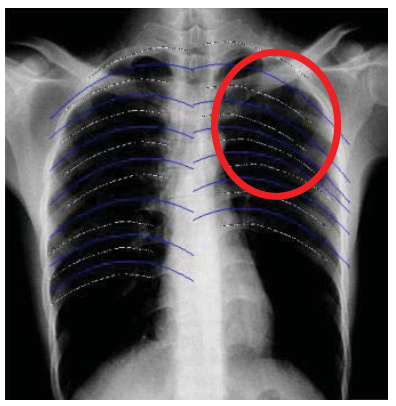

(b) ribs not clear

FIGURE 10. incorrect locating cases

\section{CONCLUSION AND FUTURE WORK}

In this work, a rib locating method using watershed algorithm and correlation matching was presented. The error rate is 0.067 and accuracy and sensitivity are respectively 0.828 and 0.862 . It is a significance work before rib segmentation. It can improve the accuracy and efficiency of rib segmentation. However, when the ribs are out of lung area or the ribs are not clear, our method may lose its effectiveness and lead to incorrect result.

The future work will focus on two aspects: 1) optimizing the rib locating method, let the model can be not only resized and rotated but reshaped to fit the ribs better and redefine the best match parameter $\mathrm{z}$ to improve the accuracy and sensitivity; 2) employing gradient based method e.g. snake or level set to extract the border of rib in each single rib area determined by rib locating method. 


\section{ACKNOWLEDGMENT}

This work has been supported by: National Program on Key Basic Research Project (No. 2013CB733800, 2013CB733803), Key Joint Program of National Natural Science Foundation and Guangdong Province (No. U1201257), and National Natural Science Foundation of China (No. 61272328), Guangdong Natural Science Foundation (No. S2011010001820). Authors would like to thank the Beijing Aerospace Zhongxing Medical Systems Co, Ltd for providing the clinical data.

\section{REFERENCES}

1. J. I. Toriwaki, Y. Suenaga, T. Negoro and T. Fukumura, "Pattern recognition of chest X-ray images," Computer Graphics and Image Processing, Vol. 2, December 1973, pp. 252-271.

2. Z. J. Yue, A. Goshtasby, L. Ackerman, "Automatic detection of rib borders in chest radiographs," IEEE Trarts. on Medical Imaging, 1995, vol. 14, pp. 525-536.

3. B. Ginneken, B. Romeny, "Automatic delineation of ribs in chest radiographs," Proceedings of SPIE, vol. 3979, pp. 825-836, 2000.

4. F. Vogelsang, M. Kohnen, J. Mahlke, etc., "Model based analysis of chest radiographs," Proceedings SPIE, vol. 3979, pp.1040-1052, 2000.

5. D. Loeckx, F. Maes, D. Vandermeulen, etc., "Temporal Subtraction of Thorax CR Images Using a Statistical Deformation Model," IEEE Transactions on Medical Imaging, vol. 22, pp.1490-1504, 2003.

6. D. H. Ballard, "Model-directed detection of ribs in chest radiographs," Proceedings of $4^{\text {th }}$ International Joint Conference on Pattern Recognition, pp. 907-910, 1978.

7. P. D. Souza, "Automatic rib detection in chest radiographs," Comput. Vis. Graph. Image Processing, vol. 23, pp. 129-161, 1983.

8. G. Powell, K. Doi, and S. Katsuragawa, "Localization of inter-rib spaces for lung texture analysis and computer-aided diagnosis in digital chest images," Med Phys., vol. 15, no. 4, pp. 581-587, 1988.

9. S. Sanada, K. Doi, and H. MacMahon, "Image feature analysis and computer-aided diagnosis in digital radiography: Automated delineation of posterior ribs in chest images," Med Phys., vol. 18, no. 5, pp. 964-971, 1991.

10. F. Meyer, "Topographic distance and watershed lines," Signal Processing, vol. 38, July 1994, pp. 113-125. 